\title{
Clinical and therapeutic significance of sirtuin-4 expression in colorectal cancer
}

\author{
GUOYU HUANG ${ }^{1,2^{*}}$, JUN CHENG $^{1 *}$, FUDONG YU $^{2}$, XISHENG LIU ${ }^{2}$, CHENWEI YUAN $^{2}$, \\ CHENCHEN LIU $^{2}$, XIAOLEI CHEN ${ }^{1}$ and ZHIHAI PENG ${ }^{2}$ \\ ${ }^{1}$ Department of Gastrointestinal Surgery, The First Affiliated Hospital, Wenzhou Medical University, \\ Wenzhou, Zhejiang 325000; ${ }^{2}$ Department of General Surgery, First People's Hospital, \\ Shanghai Jiao Tong University, Shanghai 200080, P.R. China
}

Received December 13, 2015; Accepted January 15, 2016

DOI: $10.3892 / o r .2016 .4685$

\begin{abstract}
Several members of the sirtuin family (SIRT1-7), which are a highly conserved family of $\mathrm{NAD}^{+}$-dependent enzymes, play an important role in tumor formation. Recent studies indicate that SIRT4 acts as a tumor suppressor by regulating glutamine metabolism. In the present study, we investigated the expression and activity of SIRT4 in colorectal cancer. Using a tissue microarray of 89 colorectal cancer cases, we found that SIRT4 was significantly downregulated in colorectal cancer tissues compared with that noted in the corresponding normal tissue $(\mathrm{P}<0.001)$. Lower SIRT4 levels were associated with worse pathological differentiation $(\mathrm{P}=0.031)$ and poorer post-operative overall survival rate $(\mathrm{P}=0.041)$. We found that SIRT4 overexpression inhibited the proliferation of colorectal cancer cells in vitro and in vivo. SIRT4 inhibited the glutamine metabolism of colorectal cancer cells and synergistically with glycolysis inhibitors induced cell death. SIRT4 also increased the sensitivity of colorectal cancer cells to chemotherapeutic drug 5-fluorouracil by inhibiting the cell cycle. Together, these results highlight the prognostic value of SIRT4 in colorectal cancer and the potential application of SIRT4 in colorectal cancer treatment.
\end{abstract}

\section{Introduction}

Colorectal cancer ranks third in worldwide incidence of malignant tumors, and fourth in terms of the cancer-related

Correspondence to: Dr Xiaolei Chen, Department of Gastrointestinal Surgery, The First Affiliated Hospital, Wenzhou Medical University, Wenzhou, Zhejiang 325000, P.R. China

E-mail: chen_xiaoleiwz@163.com

Dr Zhihai Peng, Department of General Surgery, First People's Hospital, Shanghai Jiao Tong University, Shanghai 200080, P.R. China

E-mail: pengzhihai.hayder@gmail.com

*Contributed equally

Key words: SIRT4, colorectal cancer, glutamine, 2-DG, 5-FU mortality rate (1). The 5-year survival rate of patients with colorectal cancer ranges between 50 and 60\% (2). Several key genes and signaling pathways were found to play an important role in the pathogenesis of colorectal cancer, for example, $E G F R, W n t, T G F \beta, p 53$ and DNA-mismatch repair pathway (3). Nonetheless, we have yet to gain a complete understanding of the molecular genetics of colorectal cancer. In the future, we also need to investigate the molecular mechanisms as well as the corresponding genetic alterations in colorectal cancer, to determine the pathophysiology and potential diagnostic markers and therapeutic targets.

The sirtuin family (SIRT1-7) includes an NAD ${ }^{+}$-dependent histone deacetylase, deacetylase and ADP ribosyltransferases playing an important role in pressure resistance, genomic stability, energy metabolism and aging (4). To date, almost all of the SIRT family members have been considered to play an important role in the development of cancer (5). In regards to SIRT1, which is the most extensively studied member of the SIRT family, the deacetylation protein group and a series of non-histone protein substrates affect the corresponding tumorrelated genes including apoptosis of FOXO proteins, tumor suppressor p53, and DNA mismatch repair protein $\mathrm{Ku} 70(6,7)$, SIRT1, SIRT3, SIRT6 and SIRT7 have been shown to play the role of oncogenes or tumor-suppressor genes in colorectal cancer (8-11). However, SIRT2, SIRT4 and SIRT5 have yet to be investigated in colorectal cancer.

SIRT2 is an NAD ${ }^{+}$-dependent deacetylase located in the cytoplasm (12), and catalyzes substrates such as H4K16 (13), H3K56 (14), FOXO1 (15) and p53 (16). Kim et al (17) found that SIRT2 regulates mitosis, and SIRT2 knockout leads to the emergence of sex-specific tumors in mice, female breast cancer and male hepatocellular carcinoma.

SIRT4 is an ADP-dependent $\mathrm{NAD}^{+}$transferase located in the mitochondria (18). SIRT4 regulates insulin secretion and fatty acid oxidation and other cellular metabolic functions (18-20). Recent studies have indicated that SIRT4 functions as a tumor-suppressor gene by regulating the metabolism of glutamine $(21,22)$.

SIRT5 is also located in the mitochondria, catalyzing deacetylation of carbamoyl-phosphate synthetase 1 (CPS1). The initial reaction of the urea cycle results in the removal and degradation of ammonia in cells (23-25). Lu et al (26) showed 
that SIRT5 expression in human non-small cell lung cancer was elevated and SIRT5 knockdown inhibits the growth and metastasis of lung cancer cells in vitro and in vivo. Therefore, we sought to determine whether SIRT2, SIRT4 and SIRT5 also play a role in colorectal cancer.

\section{Materials and methods}

Patients and colorectal cancer specimens. Tissue specimens from 16 colorectal cancer patients (age range, 45-78 years; average age, 58 years) were used in the PCR analysis. Patients underwent radical surgery for colorectal cancer at Shanghai First People's Hospital from January 2013 to May 2013. No patient received neoadjuvant chemotherapy. Patients were pathologically diagnosed with only a single primary lesion of colorectal cancer. The patients included in the present study provided written (signed) informed consent. The research protocol was approved by the Ethics Committee of Shanghai First People's Hospital. Colorectal cancer and normal colorectal tissues were removed from a $5 \mathrm{~cm}$ tumor edge, frozen with liquid nitrogen and stored at $-80^{\circ} \mathrm{C}$ until further use.

Reverse transcription-RT-PCR. Total tissue RNA was purified using the TRIzol kit (Invitrogen, Carlsbad, CA, USA) following the manufacturer's protocol. Total cDNA (500 ng) was synthesized using reverse transcription kit (PrimeScript ${ }^{\mathrm{TM}}$ RT Master Mix; Takara, Japan). The cDNA was diluted three times using an RT-PCR kit (SYBR ${ }^{\circledR}$ Premix Ex Taq ${ }^{\mathrm{TM}}$ II; Takara) in the RT-PCR reaction apparatus (DNA Engine Opticon 2 system; Bio-Rad, Hercules, CA, USA). GAPDH was selected as the reference gene. Primers for each gene were as follows: SIRT2 forward primer, ATAACCCACACCCAGCGTAG and reverse primer, AATGTCTTCTGCCCATCCAG; SIRT4 forward primer, GATGACTTGGCGTGTCTGAA and reverse primer, TTGAATGGGAACTGGAATCTG; SIRT5 forward primer, TTGAATGGGAACTGGAATCTG and reverse primer, TTG AATGGGAACTGGAATCTG; and GAPDH forward primer, CGGAGTCAACGGATTTGGTCGTAT and reverse primer, AGCCTTCTCCATGGTGGTGAAGAC. The PCR reaction conditions were as follows: $2 \mathrm{~min}$ at $94^{\circ} \mathrm{C}$ and then $30 \mathrm{sec}$ at $94^{\circ} \mathrm{C}, 30 \mathrm{sec}$ at $57^{\circ} \mathrm{C}, 1 \mathrm{~min}$ at $72^{\circ} \mathrm{C}$ for 40 cycles, and $5 \mathrm{~min}$ at $72^{\circ} \mathrm{C}$ and maintained at $4^{\circ} \mathrm{C}$. After the loop, melting curve was analyzed to ensure uniformity of the PCR product. The data were converted using the $2^{-\Delta \Delta C t}$ method.

Analysis of SIRT2, SIRT4 and SIRT5 expression using an online microarray database. The human colorectal cancer expression microarray data downloaded from the The Cancer Genome Atlas (TCGA) website (http://cancergenome.nih. gov/) were used to analyze the mRNA expression of SIRT2, SIRT4 and SIRT5 between normal colorectal and colorectal cancer tissues.

Tissue microarray. Tissue microarray was obtained from a commercial chip Co. (Superchip Inc., Shanghai, China) using 89 cases of patient samples, each containing colorectal cancer and the corresponding normal colorectal tissue specimen at each point. The point diameter was $1.5 \mathrm{~mm}$, and all points were overlaid with paraffin wax. No patient received neoadjuvant chemotherapy or radiotherapy. Surgeries were conducted between January 2009 and October 2009. The follow-up time ranged from 4.65 to 5.3 years, ending May 2014. The total survival time was defined as the time until death following radical surgery. Clinicopathological parameters included age, gender, tumor size, growth mode, degree of differentiation, tumor invasion depth and scope, lymph node and distant metastases, the Union for International Cancer Control (UICC) stage and post-operative overall survival time (OS) (Table II).

Immunohistochemistry (IHC). IHC was performed as previously described (27). SIRT4 immunoreactivity at each tissue point was evaluated in terms of staining intensity $(0$, no staining; 1 , weak staining; 2 , medium staining; and 3, strong staining), and staining area $(0,<5 \% ; 1,5-25 \% ; 2,>25-50 \%$; $3,>50-75 \%$; and $4,>75 \%$ ). The staining intensity score was multiplied with the staining area score to obtain the final staining score. The tissue points were divided into two groups based on the final staining score: low, 0-4; high, 6-12. In case of inconsistencies, the scoring was reevaluated by two researchers using a multi-headed microscope until a conclusion or consensus was reached.

Vector and virus production. A lentivirus for SIRT4 overexpression was purchased from HanBio (Shanghai, China). The virus vector was pHBLV-CMVIE-Zs Green-T2A-Puro. The final virus titer of the overexpressing lentivirus and the negative control virus was $2 \times 10^{8} \mathrm{PFU} / \mathrm{ml}$.

Cell lines and culture conditions. Human colorectal cancer cell lines RKO and HT29 were purchased from Shanghai Institute of Cell Biology, Chinese Academy of Sciences. The cells were maintained in Dulbecco's modified Eagle's medium (DMEM) supplemented with 10\% fetal bovine serum (FBS) and penicillin/streptomycin (Gibco, Grand Island, NY, USA) and incubated at $37^{\circ} \mathrm{C}$ and $5 \% \mathrm{CO}_{2}$. The stable cell line with SIRT4 overexpression was transfected with the lentivirus and screened with puromycin $(2 \mu \mathrm{g} / \mathrm{ml})$ for two weeks. The other reagents used in the cell experiments were: DMEM without glucose, DMEM (both from Gibco) without glutamine, DM-KG (349631), 2-DG (Klamar; 154-17-6) and 5-fluorouracil (5-FU) (F6627) (all from Sigma, St. Louis, MO, USA). None of the culture media contained sodium pyruvate.

Cell proliferation activity and toxicity. Cells were seeded at 1,000/well for cell proliferation activity and 5,000/well for cell proliferation toxicity into a $96-$ well plate. For detection, each well was supplemented with $10 \mu$ l Cell Counting Kit-8 (CCK-8) (Dojindo, Japan) solution, and the absorbance was read at $450 \mathrm{~nm}$ after culturing in a $\mathrm{CO}_{2}$ incubator for $2 \mathrm{~h}$. The cell proliferation activity in media without glutamine or glucose was tested by changing the media to the corresponding experimental conditions on the second day after seeding the cells. For cell proliferation toxicity, the media were replaced the next day with different doses of 5-FU. The cell proliferation toxicity was calculated dynamically: cell viability $(\%)=\mathrm{A}_{450}$ of treated cells $/ \mathrm{A}_{450}$ of untreated cells. Statistical analysis of cell proliferation toxicity was carried out using the cell viability $(\%)$ in three independent experiments. 
Table I. SIRT4 protein expression in colorectal cancer and adjacent normal colon tissues.

\begin{tabular}{|c|c|c|c|c|c|}
\hline & \multirow[b]{2}{*}{ All cases } & \multicolumn{2}{|c|}{ SIRT4 expression } & \multirow[b]{2}{*}{$\chi^{2}$} & \multirow[b]{2}{*}{ P-value } \\
\hline & & Low $(\%)$ & High (\%) & & \\
\hline Tissue type & & & & 18.574 & 0.000 \\
\hline Normal & 89 & $8(8.99)$ & $81(91.01)$ & & \\
\hline Cancer & 89 & $32(35.96)$ & $57(64.04)$ & & \\
\hline
\end{tabular}

Bold values are statistically significant $(\mathrm{P}<0.05) .{ }^{\mathrm{a}} \mathrm{Chi}$-square test.

Clone formation assay. The cells were seeded at 200/well in 6-well plates, changing the liquid every other day. After culturing for 2 weeks, the number of clones was counted directly with the naked eye after fixing with methanol and staining by Giemsa.

Flow cytometric analysis of apoptosis and cell cycle. Cells were harvested by trypsinization, pelleted by centrifugation and resuspended in phosphate-buffered saline (PBS) containing 3\% FBS. Early cellular apoptosis was measured by flow cytometry (C6) using Annexin V-APC and 7-AAD staining with Accuri C6 software (all from BD, USA). The survival rate was calculated using unstained APC or 7-AAD and found to be $100 \%$. Cell cycle was measured with PI/Nase kit (BD) according to the manufacturer's instructions. The cell cycle results were analyzed using the software ModFit (Verity Software House, Topsham, ME, USA).

Western blotting. Cells were lysed with RIPA lysis buffer supplemented with protease inhibitor cocktail (both from Beyotime, China). Protein concentrations were determined using the BCA protein concentration reagent kit (Beyotime). Cell lysates were separated by SDS-PAGE and transferred to PVDF membranes. Antibodies used were: rabbit anti-human SIRT4 polyclonal antibody (HPA029692; Sigma), goat antirabbit antibody (ab97200) and rabbit anti-human $\beta$-actin polyclonal antibody (ab11971) (both from Abcam, Cambridge, UK).

Xenograft tumorigenesis. Eight 4-week-old male BALB/c nude mice were obtained from Shanghai SLAC Laboratory Animal Co., Ltd. (SLAC; China) and bred under specific pathogen-free conditions. All animal studies were conducted in accordance with the NIH animal use guidelines and current Chinese regulations and standards for laboratory animal use. Vector and SIRT-OE RKO cells were resuspended in DMEM containing $10 \%$ FBS. The cell suspension was pre-cooled on ice before bilateral inguinal subcutaneous injection, into each mouse with an equal number of RKO cells $\left(5 \times 10^{6}\right)$, in a volume of $\sim 200 \mu 1$, on the left side of the negative vector group, and the right side in the SIRT-OE group. Two months after injection, the mice were sacrificed and the tumor was weighed.

Statistical analysis. Statistical analysis was performed using the SPSS 20.0 version of the statistical software. PCR analysis of 16 paired human colorectal cancer was followed by t-test comparing adjacent normal colorectal tissues and the proliferation toxicity of the colorectal cancer cells to 5-FU. Card and Fisher's exact tests were used to analyze the SIRT4 expression in the tumor and matched non-tumor tissues, and the SIRT4 expression in relation to clinical and pathological parameters in colorectal cancer. Kaplan-Meier analysis (the log-rank test) was used for single factor analysis. Cox proportional hazards regression model was used to identify independent prognostic factors. Other experiments were analyzed by the non-paired t-test. A P-value of $<0.05$ (two-tailed) was considered statistically significant.

\section{Results}

Decreased SIRT4 mRNA expression. We compared the mRNA expression levels of SIRT2, SIRT4 and SIRT5 in 16 paired colorectal cancer and adjacent normal tissues and found that SIRT4 was significantly reduced in colorectal cancer, while SIRT2 and SIRT5 showed no significant change (Fig. 1A). Although not statistically significant, the expression of SIRT5 in colorectal cancer showed a downward trend.

To validate the above results, we further analyzed the expression profile of SIRT2, SIRT4 and SIRT5 using microarray data, with 236 cases of colorectal cancer and 22 normal colorectal tissue samples from the TCGA database. Consistent with our RT-PCR results, we found that SIRT4 was downregulated in early stages and, importantly, its low expression was maintained during cancer progression, indicating that SIRT4 downregulation may be required for both tumor initiation and maintenance. The SIRT2 mRNA expression was downregulated in stages II and IV with no significant changes in stages I and III. SIRT5 showed no significant change in any colorectal cancer stage (Fig. 1B). In brief, the SIRT4 mRNA expression in colorectal cancer tissues was significantly decreased.

SIRT4 expression correlates with pathological differentiation and prognosis. We next evaluated SIRT4 protein expression in tissue microarray analysis of 89 colorectal cancer patients by immunohistochemistry. We observed that SIRT4 was expressed in the cytoplasm (Fig. 2). We then divided the samples into two groups defined as low and high expression based on the staining results. We found that in normal colorectal tissues, $91.01 \%$ (81/89) of the SIRT4 segment was highly expressed and $8.99 \%$ (8/89) were low. By contrast, in 

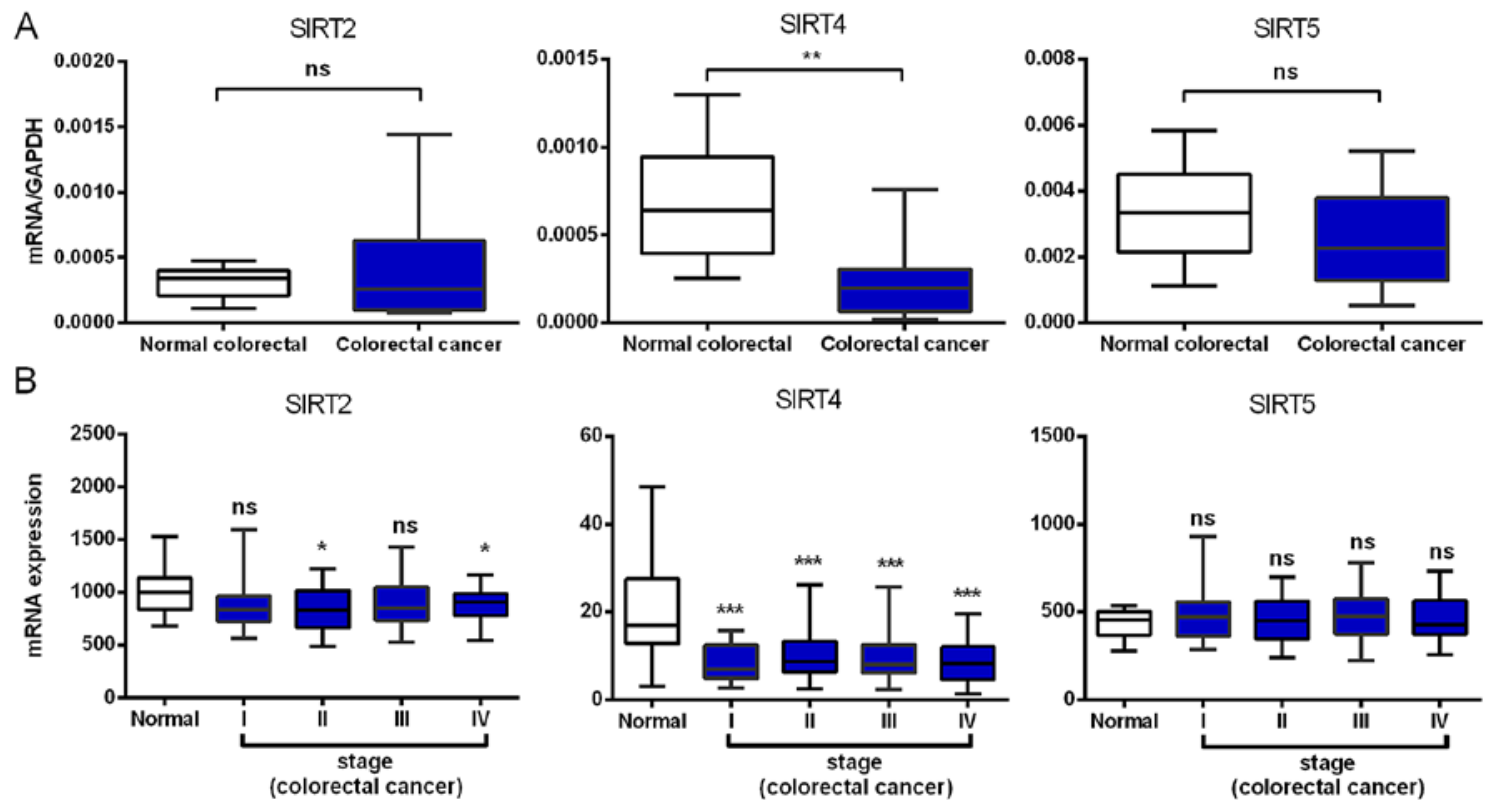

Figure 1. SIRT2, SIRT4 and SIRT5 mRNA expression in human colorectal cancer and normal colorectal tissues. (A) qRT-PCR analysis of SIRT2, SIRT4 and SIRT5 mRNA expression in 16 paired colorectal cancer and adjacent normal cells, using GAPDH as the internal reference. (B) SIRT2, SIRT4 and SIRT5 mRNA expression in colorectal cancer microarray (data download from TCGA database). Data are means \pm SEM. The boxes represent the interquartile range; whiskers represent the 5-95th percentile range; bars represent the median; ${ }^{*} \mathrm{P}<0.05,{ }^{* *} \mathrm{P}<0.01,{ }^{* * *} \mathrm{P}<0.001$.
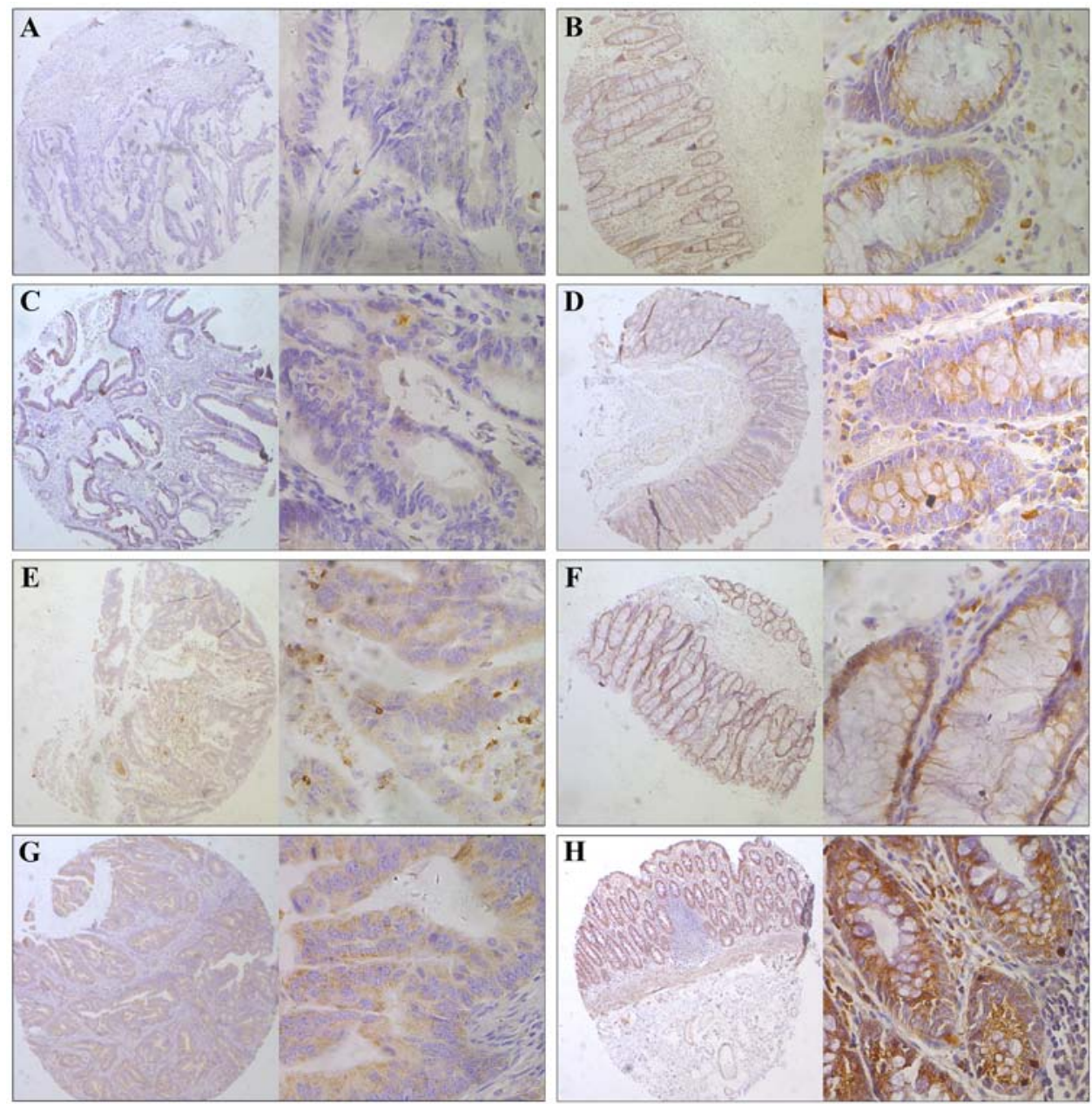

Figure 2. Representative immunohistochemical staining of SIRT4 in human colorectal cancer tissues. SIRT4 expression in the cytoplasm was significantly lower in tumor tissues compared with that observed in the adjacent normal colorectal tissue. The micrographs showed negative (A), weak (C), medium (E) and strong (G) expression of SIRT4 in colorectal cancer tissues The relevant SIRT4 expression in corresponding adjacent normal colorectal tissues of cases in A, $\mathrm{C}, \mathrm{E}$, and $\mathrm{G}$ is shown in $\mathrm{B}, \mathrm{D}, \mathrm{F}$ and $\mathrm{H}$, respectively (magnification: left panel, $\mathrm{x} 100$; right panel, $\mathrm{x} 400$ ). 
Table II. Correlation between the clinicopathologic variables and SIRT4 expression in colorectal cancer.

\begin{tabular}{|c|c|c|c|c|c|}
\hline \multirow{2}{*}{$\begin{array}{l}\text { Clinicopathological } \\
\text { parameters }\end{array}$} & \multirow{2}{*}{$\begin{array}{c}\text { All } \\
\text { cases }\end{array}$} & \multicolumn{2}{|c|}{ IRT4 expression } & \multirow[b]{2}{*}{$\chi^{2}$} & \multirow[b]{2}{*}{ P-value ${ }^{a}$} \\
\hline & & Low & High & & \\
\hline Age (years) & & & & 1.435 & 0.263 \\
\hline$\leq 65$ & 41 & 16 & 25 & & \\
\hline$>65$ & 48 & 13 & 35 & & \\
\hline Gender & & & & 0.829 & 0.377 \\
\hline Male & 46 & 17 & 29 & & \\
\hline Female & 43 & 12 & 31 & & \\
\hline Tumor size $(\mathrm{cm})$ & & & & 0.113 & 0.820 \\
\hline$\leq 5$ & 53 & 18 & 35 & & \\
\hline$>5$ & 36 & 11 & 25 & & \\
\hline Differentiation & & & & 5.791 & 0.031 \\
\hline Well-moderate & 75 & 23 & 52 & & \\
\hline Poor & 14 & 9 & 5 & & \\
\hline Stage $(\mathrm{T})$ & & & & 3.308 & 0.326 \\
\hline $\mathrm{T} 1$ & 3 & 0 & 3 & & \\
\hline $\mathrm{T} 2$ & 10 & 3 & 7 & & \\
\hline T3 & 49 & 16 & 33 & & \\
\hline $\mathrm{T} 4$ & 27 & 13 & 14 & & \\
\hline Stage (N) & & & & 1.800 & 0.475 \\
\hline No & 58 & 22 & 36 & & \\
\hline N1 & 23 & 6 & 17 & & \\
\hline $\mathrm{N} 2$ & 8 & 4 & 4 & & \\
\hline Stage (M) & & & & 0.009 & 1.000 \\
\hline M0 & 86 & 31 & 55 & & \\
\hline M1 & 3 & 1 & 2 & & \\
\hline UICC stage & & & & 2.381 & 0.478 \\
\hline I & 11 & 2 & 9 & & \\
\hline II & 45 & 19 & 26 & & \\
\hline III & 30 & 10 & 20 & & \\
\hline IV & 3 & 1 & 2 & & \\
\hline
\end{tabular}

Bold values are statistically significant $(\mathrm{P}<0.05)$. ${ }^{\mathrm{a}}$ Chi-square test. UICC, Union for International Cancer Control.

colorectal cancer tissues, these numbers were $64.04 \%(57 / 89)$ and $35.96 \%$ (32/89), respectively. The difference was statistically significant $(\mathrm{P}<0.001$; Table I).

We next analyzed the relationship between SIRT4 expression and the clinicopathological parameters and found that patients expressing low SIRT4 levels manifested increasingly adverse pathological grade $(\mathrm{P}=0.031)$. However, we did not find any statistical relationship between SIRT4 expression with other parameters, including age, gender, tumor size, tumor invasion depth $(\mathrm{T})$, lymph node positive number $(\mathrm{N})$, distant metastasis $(\mathrm{M})$ and UICC stage $(\mathrm{P}>0.05)$. The relationship between SIRT4 expression and the clinicopathological is summarized in Table II.

To further explore the prognostic value of SIRT4 in colorectal cancer, we first performed univariate analysis. The
Table III. Univariate analysis of SIRT4 expression and clinicopathological variables in 89 patients with colorectal cancer.

\begin{tabular}{|c|c|c|c|c|}
\hline \multirow[b]{2}{*}{ Variable } & \multirow[b]{2}{*}{ All cases } & \multicolumn{2}{|c|}{$\begin{array}{l}\text { Overall survival } \\
\quad \text { (months) }\end{array}$} & \multirow[b]{2}{*}{ P-value ${ }^{a}$} \\
\hline & & Mean & Median & \\
\hline Age (years) & & & & 0.531 \\
\hline$\leq 65$ & 41 & 46.6 & NR & \\
\hline$>65$ & 48 & 49.3 & NR & \\
\hline Gender & & & & 0.368 \\
\hline Male & 46 & 49.3 & NR & \\
\hline Female & 43 & 46.7 & NR & \\
\hline Tumor size $(\mathrm{cm})$ & & & & 0.005 \\
\hline$\leq 5$ & 53 & 54.2 & NR & \\
\hline$>5$ & 36 & 38.9 & 38 & \\
\hline Differentiation & & & & 0.003 \\
\hline Well-moderate & 75 & 51.1 & NR & \\
\hline Poor & 14 & 30.6 & 17 & \\
\hline T stage & & & & 0.247 \\
\hline $\mathrm{T} 1-\mathrm{T} 2$ & 13 & 52.6 & NR & \\
\hline T3-T4 & 76 & 47.0 & NR & \\
\hline N stage & & & & 0.000 \\
\hline No & 58 & 53.7 & NR & \\
\hline $\mathrm{N} 1-\mathrm{N} 2$ & 31 & 37.1 & 39 & \\
\hline M stage & & & & 0.002 \\
\hline M0 & 86 & 49.1 & NR & \\
\hline M1 & 3 & 18.3 & 17 & \\
\hline UICC stage & & & & 0.000 \\
\hline I-II & 56 & 54.9 & NR & \\
\hline III-IV & 33 & 36.0 & 25 & \\
\hline SIRT4 expression & & & & 0.041 \\
\hline Low & 32 & 40.3 & 44 & \\
\hline High & 57 & 51.7 & NR & \\
\hline
\end{tabular}

Bold values are statistically significant $(\mathrm{P}<0.05)$. NR, not reached; UICC, Union for International Cancer Control. ${ }^{a}$ Log-rank test.

results showed that SIRT4 and tumor size, tumor differentiation, lymph node and tumor distant metastasis, and UICC stage were related to OS time post-operatively (Table III). The OS of patients with low SIRT4 expression was significantly lower than that noted in patients with high SIRT4 expression ( $\mathrm{P}=0.041$, test log-rank; Fig. 3). Next, using COX regression analysis adjusted for the prognostic factors established in the univariate analysis, we found a significant correlation between low SIRT4 expression and worse OS time of the colorectal cancer patients $(\mathrm{P}=0.003, \mathrm{HR}=0.339$; Table IV $)$. Together, these results suggest that SIRT4 expression is associated with a worse pathological grade and is an independent prognostic factor for OS in patients with colorectal cancer.

SIRT4 inhibits the growth of human colorectal cancer cells. We constructed a stable cell line overexpressing SIRT4 in 
Table IV. Cox multivariate analyses of prognostic factors on overall survival.

\begin{tabular}{lccc}
\hline Variables & HR & $95 \%$ CI & P-value $^{\mathrm{a}}$ \\
\hline Tumor size (cm) ( $\leq 5$ vs. $>5$ ) & 2.781 & $1.384-5.590$ & $\mathbf{0 . 0 0 4}$ \\
Differentiation (Well/moderate vs. poor) & & & NS \\
N stage (N0 vs. N1/N2) & & & NS \\
M stage (M0 vs. M1) & 4.555 & $2.201-9.426$ & NS \\
UICC stage (I/II vs. III/IV) & 0.339 & $0.165-0.695$ & $\mathbf{0 . 0 0 0}$ \\
SIRT4 expression (Low vs. High) & & $\mathbf{0 . 0 0 3}$ \\
\hline
\end{tabular}

Bold values are statistically significant $(\mathrm{P}<0.05)$. HR, hazard ratio; CI, confidence interval; NS, not significant. ${ }^{\mathrm{a} F o r w a r d ~ L R ~ m e t h o d . ~}$

colorectal cancer lines RKO and HT29 using the lentivirus, and verified the results by western blotting (Fig. 4A). We found that SIRT4 overexpression significantly reduced the proliferation of RKO and HT29 cells (Fig. 4B and C). Furthermore, SIRT4 overexpression significantly reduced the number and size of the clones of RKO and HT29 cells (Fig. 4D). Next, we found that SIRT4 overexpression significantly reduced the tumorigenic potential of RKO cells in nude mice (Fig. 4E). Together, these results indicate that SIRT4 inhibits the growth of colorectal cancer cells.

We found no significant change in the apoptosis rate and cell cycle of the RKO and HT29 cells following SIRT4 overexpression (Fig. 4F and G).

SIRT4 inhibits glutamine metabolism and synergistically with glycolytic inhibition induces cell death in colorectal cancer. Studies indicate that SIRT4 inhibits tumor growth via inhibition of mitochondrial glutamine metabolism $(21,22)$. We investigated whether SIRT4 inhibited the growth of colon cancer cells by inhibiting glutamine metabolism. We found that RKO and HT29 cells still maintained growth in media in the absence of glucose, but the growth rate was significantly weak in the absence of glutamine (Fig. 5A and B) suggesting that glutamine metabolism plays an important role in the growth of human colorectal cancer cells.

We tested whether SIRT4 inhibited the utilization of glutamine in colorectal cancer cells. We deprived RKO and HT29 cells of glucose, and forced the cells to switch to glutamine to maintain growth. The results showed that SIRT4 overexpression significantly reduced the survival rate of the RKO and HT29 cells in glucose-deprivation. However, when cell-permeable DM-KG was added, the mortality difference disappeared (Fig. 5C and D). We found that in glucose-deprived media, the magnitude of the decrease in proliferation of the colorectal cancer cells caused by SIRT4 overexpression was larger compared with glucosesupplemented media (data not shown). These results indicate that SIRT4 overexpression reduced glutamine dependence of colorectal cancer cells, suggesting that SIRT4 inhibition of glutamine metabolism mediated the inhibition of proliferation of the colorectal cancer cells.

Blocking tumor cells in the metabolic pathway is a new treatment strategy. Since SIRT4 inhibited glutamine metabolism, we further explored whether SIRT4 overexpression increased the sensitivity of colorectal cancer cells to glucose metabolic inhibitors. Consistent with the previous glucose deprivation experiments, we found that SIRT4 overexpression sensitized colorectal cancer cells to 2-deoxyglucose (2-DG)-induced cell death (Fig. 5E and F) further supporting the role of SIRT4 in glutamine metabolism and survival of colorectal cells and indicating that SIRT4 overexpression and glucose metabolism inhibitors induced a synergistic effect on the colorectal cancer cells.

In addition to inhibiting glutamine metabolism, the role of SIRT4 in glucose metabolism is still unclear. We found that SIRT4 overexpression significantly reduced the survival rates of both colorectal cancer cell lines in glutamine-deprived media (Fig. 5G and $\mathrm{H}$ ) suggesting that SIRT4 affected glucose metabolism as well.

SIRT4 increases the sensitivity of colorectal cancer cells to 5-FU by delaying the cell cycle. 5-FU is the most commonly used chemotherapeutic agent for the treatment of colorectal cancer. We found that SIRT4 overexpression increased the inhibitory effect of 5-FU on the proliferation of colorectal cancer cells (Fig. 6A and B).

We found that SIRT4 overexpression significantly decreased the $\mathrm{S}$ and $\mathrm{G} 2 / \mathrm{M}$ rates in both colorectal cancer cell lines after 5-FU treatment (Fig. 6E), but had no influence on the apoptotic rates under these conditions (Fig. 6F) suggesting that SIRT4 increased the sensitivity of colorectal cancer cells to 5 -FU by delaying mitosis.

\section{Discussion}

In the present study, we found that decreased SIRT4 expression in human colorectal cancer was associated with poor pathologic differentiation and worse prognosis. In vitro and in vivo experiments demonstrated that SIRT4 decreased the proliferation activity, the number of cells and tumor formation in nude mice injected with colorectal cancer cells. We found that glutamine plays an important role in the growth of colorectal cancer cells, and SIRT4 weakened the ability of colorectal cancer cells in glutamine utilization and enhanced cell death caused by glucose metabolism inhibitor 2-DG. Finally, we found that SIRT4 increased the sensitivity of colorectal cancer cells to chemotherapeutic agents by delaying the cell cycle. Our research has uncovered the clinical significance of SIRT4 in human colon cancer. 


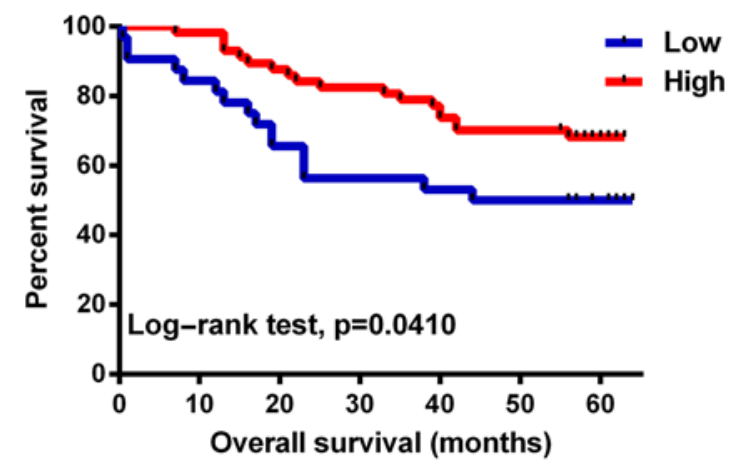

$\begin{array}{llllllllc}\begin{array}{l}\text { No. at risk } \\ \text { Low: }\end{array} & 32 & 27 & 21 & 18 & 17 & 16 & 16 & 16 \\ \text { High: } & 57 & 56 & 50 & 47 & 44 & 40 & 39 & 18\end{array}$

Figure 3. Kaplan-Meier curve comparing time to survival between colorectal cancers with low vs. high SIRT4 expression, determined using tissue microarray. The total survival rate of patients with colorectal cancer with low SIRT4 expression was significantly lower than that of SIRT4 with high expression; P=0.0410, log-rank test.
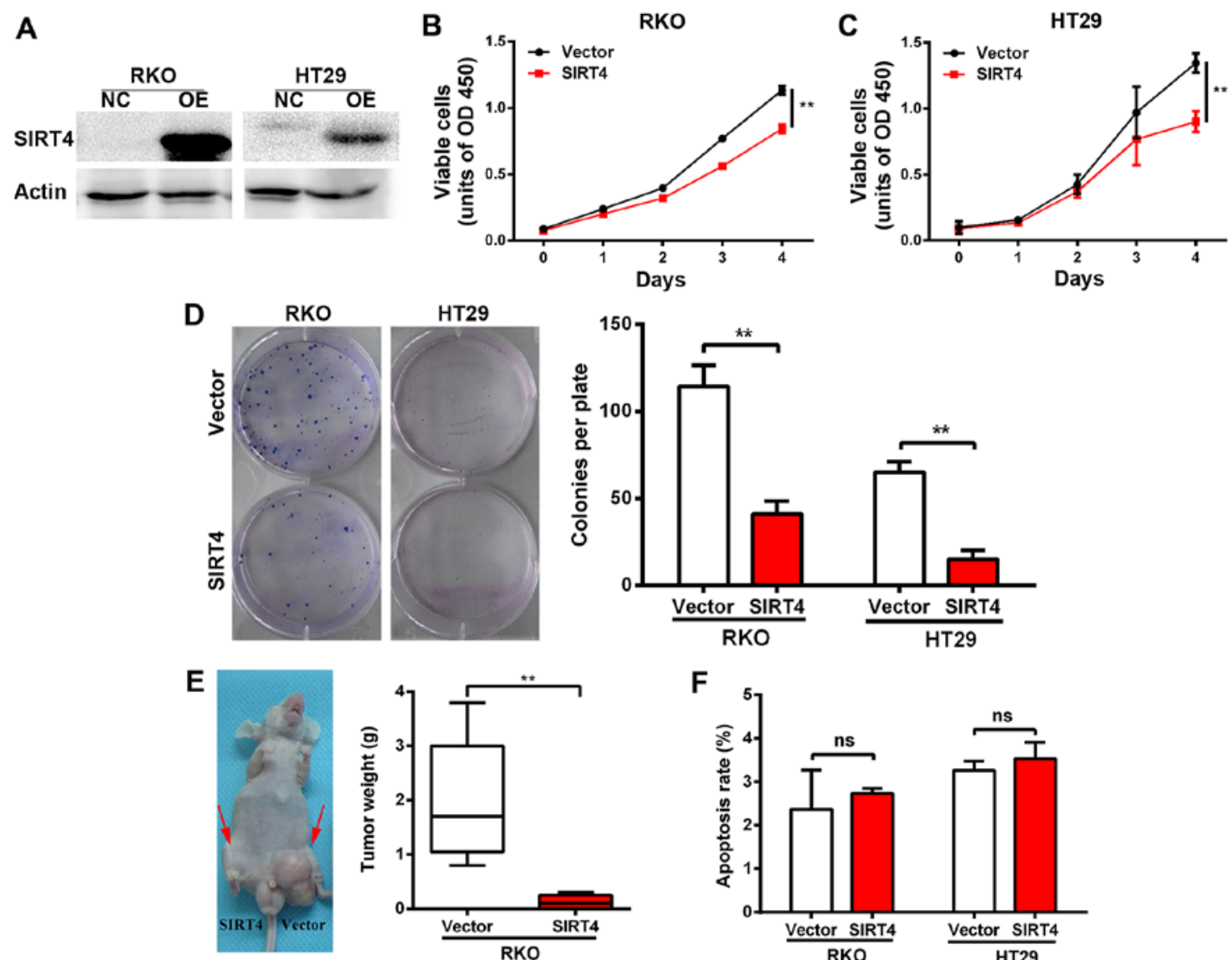

$\mathbf{F}$

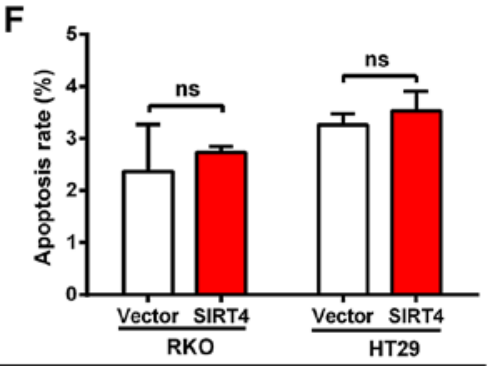

\begin{tabular}{|c|c|c|c|c|}
\hline & & G1 & $S$ & $\mathrm{G} 2 / \mathrm{M}$ \\
\hline \multirow[t]{2}{*}{ RKO } & Vector & $41.92 \pm 0.27$ & $50.08 \pm 0.27$ & $8.00 \pm 0.00$ \\
\hline & SIRT4 & $39.32 \pm 0.13$ & $52.68 \pm 0.13$ & $8.00 \pm 0.00$ \\
\hline \multirow[t]{2}{*}{ HT29 } & Vector & $47.00 \pm 1.70$ & $45.00 \pm 1.70$ & $8.00 \pm 0.00$ \\
\hline & SIRT4 & $47.37 \pm 2.74$ & $44.63 \pm 2.74$ & $8.00 \pm 0.00$ \\
\hline
\end{tabular}

Figure 4. SIRT4 inhibits the growth of colorectal cancer cells but not apoptosis or the cell cycle. (A) Western blotting of SIRT4 overexpression after treatment with puromycin $2 \mu \mathrm{g} / \mathrm{ml}$ for two weeks, using $\beta$-actin as an internal control. (B and C) Proliferation activity curve of the vector and SIRT4-OE RKO (B) and HT29 (C) cells. Cell proliferation activity was measured every $24 \mathrm{~h}$ for 4 consecutive days. (D) Representative image of the clone formation experiment using vector and SIRT4-OE RKO (right) and HT29 cells (left). Cells were stained with Giemsa and cultured for 14 days. (E) RKO cells in nude mice (left). Mice were sacrificed after two months of cell inoculation, and the tumors were removed and weighed (right). The boxes represent the interquartile range; whiskers represent the 5th-95th percentile range; bars represent the median. (F) Apoptotic rate of the vector and SIRT4-OE RKO and HT29 cells. (G) Cell cycle distribution of the vector and SIRT4-OE RKO and HT29 cells. Data are expressed as mean \pm standard deviation. ns, no significant difference; ${ }^{* *} \mathrm{P}<0.01$. 

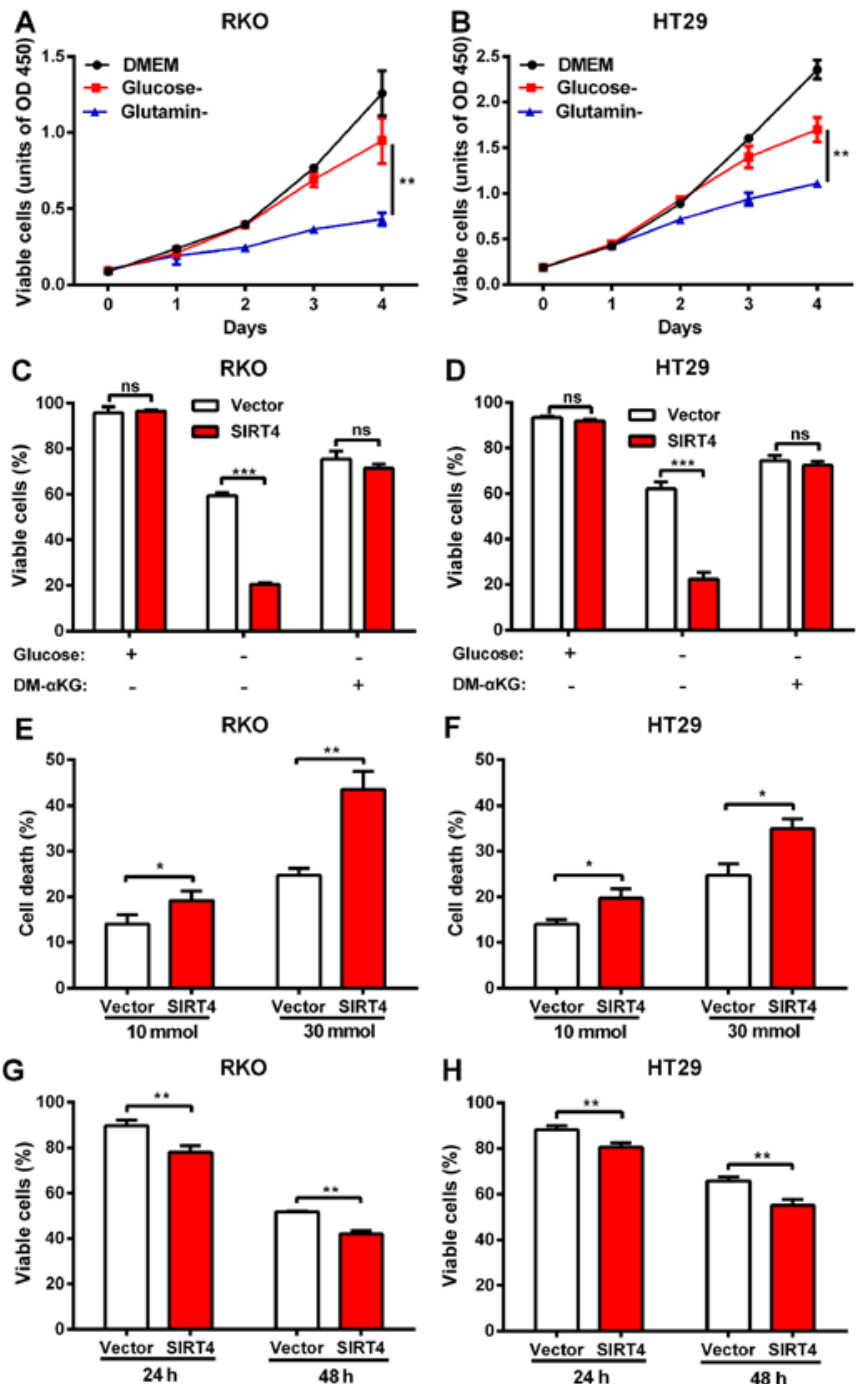

$\mathbf{F}$
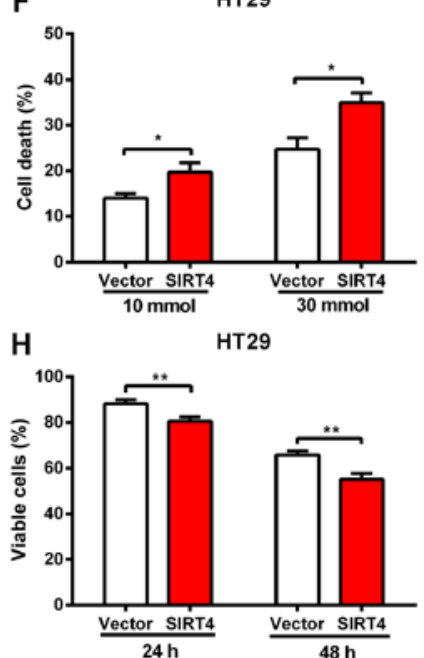

Figure 5. SIRT4 inhibits glutamine metabolism and synergizes with glycolytic inhibition to induce cell death of colorectal cancer cells. (A and B) The proliferation activity curve of RKO and HT29 cells cultured in high-glucose, glucose-deprived and glutamine-deprived DMEM. (C and D) Survival rate of vector and SIRT4-OE RKO and HT29 cells in high-glucose, glucose-deprived or glucose-deprived and supplemented with DM-KG $(7 \mathrm{mM})$ media for $48 \mathrm{~h}$. (E and F) Death rate of vector and SIRT4-OE RKO and HT29 cells after administration of the indicated doses of 2DG (10 and $30 \mathrm{mmol} / \mathrm{l})$ for $48 \mathrm{~h}$. $(\mathrm{G}$ and $\mathrm{H}$ ) Survival rate of the vector and SIRT4-OE RKO and HT29 cells in glutamine-deprived media. Data are expressed as mean \pm standard deviation; ${ }^{*} \mathrm{P}<0.05,{ }^{* *} \mathrm{P}<0.01$.

According to the present study, multiple SIRT family members are involved in different tumors, which may depend on the specific tissue and tumor type (28). For instance, SIRT1 expression levels are elevated in gastric (29), colorectal (30), prostate (31) and skin cancers (32), suggesting that it promotes tumor formation. In addition, studies have shown that SIRT1 may act as a tumor suppressor. For example, SIRT1 was downregulated in breast cancer (33) and inhibited the formation of intestinal tumors in APC $\left(\mathrm{Min} /{ }^{+}\right)$mouse models (34). Similarly, SIRT2 was found to be downregulated in breast (17), glioma (35) and skin cancers (36), but upregulated in acute myeloid leukemia (37) and prostate cancer $(38)$. Jeong et al $(21,39)$ found that SIRT4 inhibited the growth of HeLa cells and MYC-induced
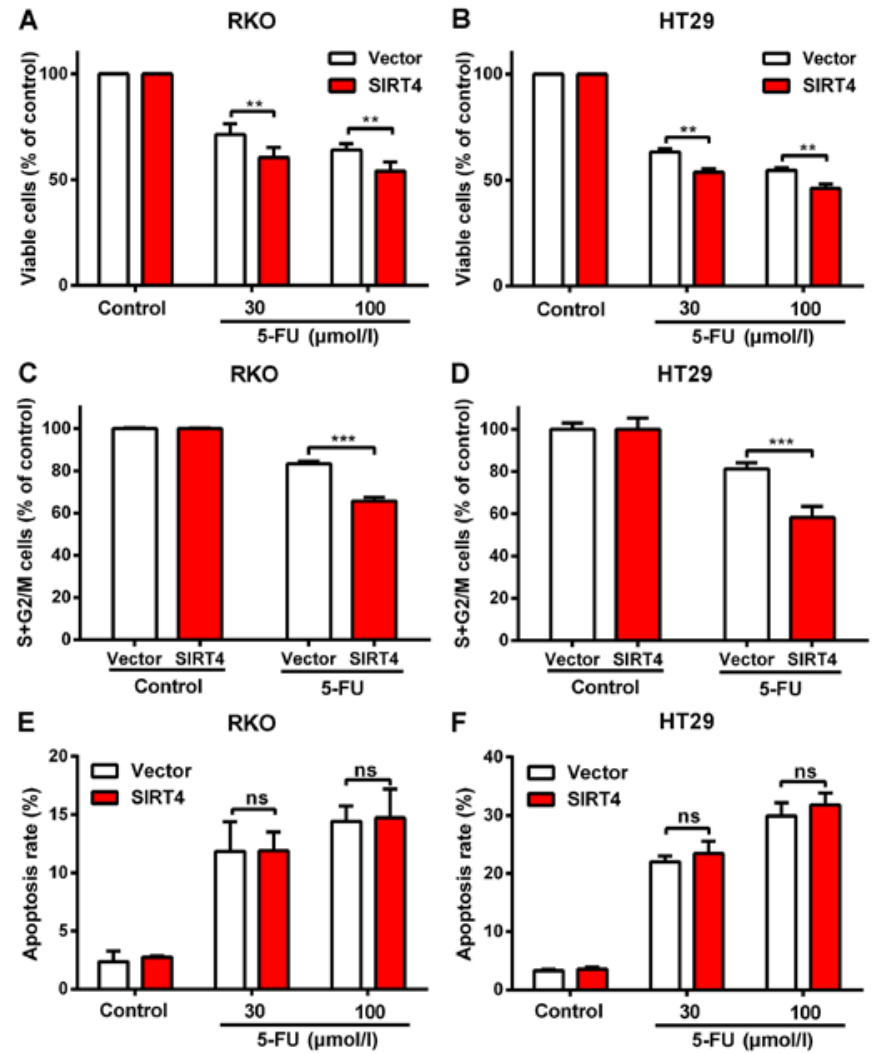

Figure 6. SIRT4 increased the sensitivity of colorectal cancer cells to chemotherapeutic drug 5-FU by delaying the cell cycle. (A and B) The cell viability of vector and SIRT4-OE RKO and HT29 cells cultured in media supplemented with 5 -FU (30 and $100 \mu \mathrm{mol} / \mathrm{l}$ ) after $24 \mathrm{~h}$ as detected by CCK- 8 reagent. Data derived from three independent experiments with triplicate wells; ${ }^{* *} \mathrm{P}<0.01$. Paired t-test. (C and D) Cell cycle of vector and SIRT4-OE RKO and HT29 cells after incubation with $30 \mu \mathrm{mol} / 15-\mathrm{FU}$ for $24 \mathrm{~h}$; ${ }^{* *} \mathrm{P}<0.01$. (E and F) The apoptotic rate of vector SIRT4-OE RKO and HT29 cells at 30 and $100 \mu \mathrm{mol} / 15$-FU. Data are expressed as mean \pm standard deviation. ns, no significant difference; ${ }^{*} \mathrm{P}<0.05,{ }^{* *} \mathrm{P}<0.01$.

B-lymphoma cells. SIRT4-knockout MEF cells in nude mice formed larger tumors. SIRT4-knockout mice spontaneously developed cancers of the lung, liver, breast and lymphoma. Csibi et al (22) also found that SIRT4 inhibited the growth of human colorectal cancer DLD-1 cells and human prostate cancer DU145 cells. Our previous studies suggested that SIRT4 expression is decreased in gastric cancer tissues and is correlated with gastric cancer pathology (27). In the present study, we found that SIRT4 was downregulated in human colorectal cancer tissues and inhibited the growth of colorectal cancer cells in vitro and in vivo. Our results suggest that SIRT4 plays a tumor-suppressor role in human colorectal cancer, and that SIRT4 is a tumor-suppressor gene.

We found no significant difference in SIRT2 and SIRT5 mRNA levels between colorectal cancer and normal colorectal tissues. Since the role of SIRT family members is tissue-specific, they do not play a role in the development of colorectal cancer. In contrast, their expression in colorectal cancer may be regulated by post transcriptional modification. SIRT1, which has a higher level of protein expression in hepatocellular carcinoma tissues compared with normal liver tissues, showed no difference in mRNA expression between 
HCC tissues and adjacent normal liver tissues (40). The next step will be to study the protein expression in colorectal cancer.

Jeong et al (21) and Csibi et al (22) found that SIRT4 inhibited tumor grow th by inhibiting mitochondrial glutamine metabolism. However, recent studies show that SIRT4 inhibits pyruvate dehydrogenase (41). Since pyruvate dehydrogenase is a key enzyme in the tricarboxylic acid cycle, SIRT4 may also play a role in glucose metabolism. The present study found that SIRT4 increased the cell death of colorectal cancer cells in glucose-deprived culture media, and the addition of glutamine to downstream metabolite DM-KG abrogated this effect suggesting that SIRT4 inhibited glutamine metabolism. However, we also found that overexpression of SIRT4 reduced the survival rate of the colorectal cancer cells in glutamine-deprived media, indicating that SIRT4 may also inhibit glucose metabolism in colorectal cancer cells.

Targeting glucose metabolism using glucose inhibitors has been used as a therapeutic strategy (42-44). However, tumor cells activate other metabolic pathways, such as glutamine metabolism to survive, since mitochondrial glutamine metabolism can substitute for the lack of glucose recharge mitochondrial tricarboxylic acid cycle $(45,46)$. Our experiments found that colorectal cancer cells under glucose deprivation still maintained growth, and SIRT4 overexpression increased the death associated with glucose deprivation in colorectal cancer. SIRT4 overexpression and glucose inhibitors 2-DG synergistically acted to significantly increase colorectal cancer cell death. These results indicate the therapeutic potential of SIRT4 targeting in metabolism, particularly in treating tumors with a glucose metabolism inhibitor.

Previous research has shown that SIRT4 delays the cell cycle in damaged DNA (21). The present study found that 5-FU increased the sensitivity of colorectal cancer cells to chemotherapy drugs by delaying the cell cycle. The present study reveals the potential of SIRT4 in chemotherapy.

In summary, our results indicate that SIRT4 plays a tumor-suppressor role and is an independent prognostic factor in colorectal cancer. SIRT4 for the treatment of colorectal cancer, particularly in conjunction with metabolic and cytotoxic chemotherapy, is a promising strategy. The present study reveals the clinical significance of SIRT4 in human colorectal cancer. Our results suggest that SIRT4 is a potential diagnostic and therapeutic target in colorectal cancer.

\section{Acknowledgements}

We thank Yueqin Tang for technical assistance in the experimental study of nude mice. We also thank Huamei Tang for assistance with the immunohistochemistry studies. The present study was funded by the National High Technology Research and Development Program (SS2014AA020803), the National Natural Science Foundation of China (81220108021), the Project of Shanghai Science and Technology Commission (14411950502), the Joint Research Projects of Shanghai Municipal Hospital (SHDC12012105), the Project of Shanghai JiaoTong University (YG2012ZD01), and the Nutriology of the Medical Support Discipline of Zhejiang Province.

\section{References}

1. Brenner H, Kloor M and Pox CP: Colorectal cancer. Lancet 383: 1490-1502, 2014.

2. Siegel R, DeSantis C, Virgo K, Stein K, Mariotto A, Smith T, Cooper D, Gansler T, Lerro C, Fedewa S, et al: Cancer treatment and survivorship statistics, 2012. CA Cancer J Clin 62: 220-241, 2012.

3. Fearon ER: Molecular genetics of colorectal cancer. Annu Rev Pathol 6: 479-507, 2011.

4. Finkel T, Deng CX and Mostoslavsky R: Recent progress in the biology and physiology of sirtuins. Nature 460: 587-591, 2009.

5. Yuan H, Su L and Chen WY: The emerging and diverse roles of sirtuins in cancer: A clinical perspective. Onco Targets Ther 6: 1399-1416, 2013.

6. Chen WY, Wang DH, Yen RC, Luo J, Gu W and Baylin SB: Tumor suppressor HIC1 directly regulates SIRT1 to modulate p53-dependent DNA-damage responses. Cell 123: 437-448, 2005.

7. Brunet A, Sweeney LB, Sturgill JF, Chua KF, Greer PL, Lin Y, Tran H, Ross SE, Mostoslavsky R, Cohen HY, et al: Stress-dependent regulation of FOXO transcription factors by the SIRT1 deacetylase. Science 303: 2011-2015, 2004.

8. Yu H, Ye W, Wu J, Meng X, Liu RY, Ying X, Zhou Y, Wang H, Pan C and Huang W: Overexpression of sirt7 exhibits oncogenic property and serves as a prognostic factor in colorectal cancer. Clin Cancer Res 20: 3434-3445, 2014.

9. Liu C, Huang Z, Jiang H and Shi F: The sirtuin 3 expression profile is associated with pathological and clinical outcomes in colon cancer patients. Biomed Res Int 2014: 871263, 2014.

10. Sebastián C, Zwaans BM, Silberman DM, Gymrek M, Goren A, Zhong L, Ram O, Truelove J, Guimaraes AR, Toiber D, et al: The histone deacetylase SIRT6 is a tumor suppressor that controls cancer metabolism. Cell 151: 1185-1199, 2012.

11. Kabra N, Li Z, Chen L, Li B, Zhang X, Wang C, Yeatman T, Coppola D and Chen J: SirT1 is an inhibitor of proliferation and tumor formation in colon cancer. J Biol Chem 284: 18210-18217, 2009.

12. North BJ, Marshall BL, Borra MT, Denu JM and Verdin E: The human Sir2 ortholog, SIRT2, is an $\mathrm{NAD}^{+}$-dependent tubulin deacetylase. Mol Cell 11: 437-444, 2003.

13. Vaquero A, Scher MB, Lee DH, Sutton A, Cheng HL, Alt FW, Serrano L, Sternglanz R and Reinberg D: SirT2 is a histone deacetylase with preference for histone $\mathrm{H} 4 \mathrm{Lys} 16$ during mitosis. Genes Dev 20: 1256-1261, 2006.

14. Das C, Lucia MS, Hansen KC and Tyler JK: CBP/p300-mediated acetylation of histone H3 on lysine 56. Nature 459: 113-117, 2009.

15. Jing E, Gesta S and Kahn CR: SIRT2 regulates adipocyte differentiation through FoxO1 acetylation/deacetylation. Cell Metab 6: 105-114, 2007.

16. Jin YH, Kim YJ, Kim DW, Baek KH, Kang BY, Yeo CY and Lee KY: Sirt2 interacts with 14-3-3 beta/gamma and down-regulates the activity of p53. Biochem Biophys Res Commun 368: 690-695, 2008

17. Kim HS, Vassilopoulos A, Wang RH, Lahusen T, Xiao Z, Xu X, Li C, Veenstra TD, Li B, Yu H, et al: SIRT2 maintains genome integrity and suppresses tumorigenesis through regulating APC/C activity. Cancer Cell 20: 487-499, 2011.

18. Haigis MC, Mostoslavsky R, Haigis KM, Fahie K, Christodoulou DC, Murphy AJ, Valenzuela DM, Yancopoulos GD, Karow M, Blander G, et al: SIRT4 inhibits glutamate dehydrogenase and opposes the effects of calorie restriction in pancreatic beta cells. Cell 126: 941-954, 2006.

19. Nasrin N, Wu X, Fortier E, Feng Y, Bare' OC, Chen S, Ren X, Wu Z, Streeper RS and Bordone L: SIRT4 regulates fatty acid oxidation and mitochondrial gene expression in liver and muscle cells. J Biol Chem 285: 31995-32002, 2010.

20. Ahuja N, Schwer B, Carobbio S, Waltregny D, North BJ, Castronovo V, Maechler P and Verdin E: Regulation of insulin secretion by SIRT4, a mitochondrial ADP-ribosyltransferase. J Biol Chem 282: 33583-33592, 2007.

21. Jeong SM, Xiao C, Finley LW, Lahusen T, Souza AL, Pierce K, Li YH, Wang X, Laurent G, German NJ, et al: SIRT4 has tumorsuppressive activity and regulates the cellular metabolic response to DNA damage by inhibiting mitochondrial glutamine metabolism. Cancer Cell 23: 450-463, 2013. 
22. Csibi A, Fendt SM, Li C, Poulogiannis G, Choo AY, Chapski DJ, Jeong SM, Dempsey JM, Parkhitko A, Morrison T, et al: The mTORC1 pathway stimulates glutamine metabolism and cell proliferation by repressing SIRT4. Cell 153: 840-854, 2013

23. Tan M, Peng C, Anderson KA, Chhoy P, Xie Z, Dai L, Park J, Chen Y, Huang H, Zhang Y, et al: Lysine glutarylation is a protein posttranslational modification regulated by SIRT5. Cell Metab 19: 605-617, 2014.

24. Du J, Zhou Y, Su X, Yu JJ, Khan S, Jiang H, Kim J, Woo J, $\mathrm{Kim} \mathrm{JH}$, Choi BH, et al: Sirt5 is a NAD-dependent protein lysine demalonylase and desuccinylase. Science 334: 806-809, 2011.

25. Nakagawa T, Lomb DJ, Haigis MC and Guarente L: SIRT5 deacetylates carbamoyl phosphate synthetase 1 and regulates the urea cycle. Cell 137: 560-570, 2009.

26. Lu W, Zuo Y, Feng Y and Zhang M: SIRT5 facilitates cancer cell growth and drug resistance in non-small cell lung cancer. Tumour Biol 35: 10699-10705, 2014.

27. Huang G, Cui F, Yu F, Lu H, Zhang M, Tang H and Peng Z: Sirtuin-4 (SIRT4) is downregulated and associated with some clinicopathological features in gastric adenocarcinoma. Biomed Pharmacother 72: 135-139, 2015.

28. Roth M and Chen WY: Sorting out functions of sirtuins in cancer. Oncogene 33: 1609-1620, 2014.

29. Cha EJ, Noh SJ, Kwon KS, Kim CY, Park BH, Park HS, Lee H, Chung MJ, Kang MJ, Lee DG, et al: Expression of DBC1 and SIRT1 is associated with poor prognosis of gastric carcinoma. Clin Cancer Res 15: 4453-4459, 2009.

30. Stünkel W, Peh BK, Tan YC, Nayagam VM, Wang X, Salto-Tellez M, Ni B, Entzeroth M and Wood J: Function of the SIRT1 protein deacetylase in cancer. Biotechnol J 2: 1360-1368, 2007.

31. Huffman DM, Grizzle WE, Bamman MM, Kim JS, Eltoum IA, Elgavish A and Nagy TR: SIRT1 is significantly elevated in mouse and human prostate cancer. Cancer Res 67: 6612-6618, 2007.

32. Hida Y, Kubo Y, Murao K and Arase S: Strong expression of a longevity-related protein, SIRT1, in Bowen's disease. Arch Dermatol Res 299: 103-106, 2007.

33. Wang RH, Sengupta K, Li C, Kim HS, Cao L, Xiao C, Kim S, $\mathrm{Xu}$ X, Zheng Y, Chilton B, et al: Impaired DNA damage response, genome instability, and tumorigenesis in SIRT1 mutant mice. Cancer Cell 14: 312-323, 2008.

34. Firestein R, Blander G, Michan S, Oberdoerffer P, Ogino S, Campbell J, Bhimavarapu A, Luikenhuis S, de Cabo R, Fuchs C, et al: The SIRT1 deacetylase suppresses intestinal tumorigenesis and colon cancer growth. PLoS One 3: e2020, 2008.
35. Hiratsuka M, Inoue T, Toda T, Kimura N, Shirayoshi Y, Kamitani H, Watanabe T, Ohama E, Tahimic CG, Kurimasa A, et al: Proteomics-based identification of differentially expressed genes in human gliomas: Down-regulation of SIRT2 gene. Biochem Biophys Res Commun 309: 558-566, 2003.

36. Ming M, Qiang L, Zhao B and He YY: Mammalian SIRT2 inhibits keratin 19 expression and is a tumor suppressor in skin. Exp Dermatol 23: 207-209, 2014.

37. Dan L, Klimenkova O, Klimiankou M, Klusman JH, van den Heuvel-Eibrink MM, Reinhardt D, Welte K and Skokowa J: The role of sirtuin 2 activation by nicotinamide phosphoribosyltransferase in the aberrant proliferation and survival of myeloid leukemia cells. Haematologica 97: 551-559, 2012.

38. Hou H, Chen W, Zhao L, Zuo Q, Zhang G, Zhang X, Wang H, Gong H, Li X, Wang M, et al: Cortactin is associated with tumour progression and poor prognosis in prostate cancer and SIRT2 other than HADC6 may work as facilitator in situ. J Clin Pathol 65: 1088-1096, 2012.

39. Jeong SM, Lee A, Lee J and Haigis MC: SIRT4 suppresses tumor formation in genetic models of Myc-induced B cell lymphoma. J Biol Chem 289: 4135-4144, 2014.

40. Chen J, Zhang B, Wong N, Lo AW, To KF, Chan AW, Ng MH, Ho CY, Cheng SH, Lai PB, et al: Sirtuin 1 is upregulated in a subset of hepatocellular carcinomas where it is essential for telomere maintenance and tumor cell growth. Cancer Res 71: 4138-4149, 2011.

41. Mathias RA, Greco TM, Oberstein A, Budayeva HG Chakrabarti R, Rowland EA, Kang Y, Shenk T and Cristea IM: Sirtuin 4 is a lipoamidase regulating pyruvate dehydrogenase complex activity. Cell 159: 1615-1625, 2014.

42. Zhao Y, Butler EB and Tan M: Targeting cellular metabolism to improve cancer therapeutics. Cell Death Dis 4: e532, 2013.

43. Galluzzi L, Kepp O, Vander Heiden MG and Kroemer G: Metabolic targets for cancer therapy. Nat Rev Drug Discov 12: 829-846, 2013.

44. Ahmad IM, Abdalla MY, Aykin-Burns N, Simons AL, Oberley LW, Domann FE and Spitz DR: 2-Deoxyglucose combined with wild-type p53 overexpression enhances cytotoxicity in human prostate cancer cells via oxidative stress. Free Radic Biol Med 44: 826-834, 2008.

45. Daye D and Wellen KE: Metabolic reprogramming in cancer: Unraveling the role of glutamine in tumorigenesis. Semin Cell Dev Biol 23: 362-369, 2012.

46. Tennant DA, Durán RV and Gottlieb E: Targeting metabolic transformation for cancer therapy. Nat Rev Cancer 10: 267-277, 2010 . 